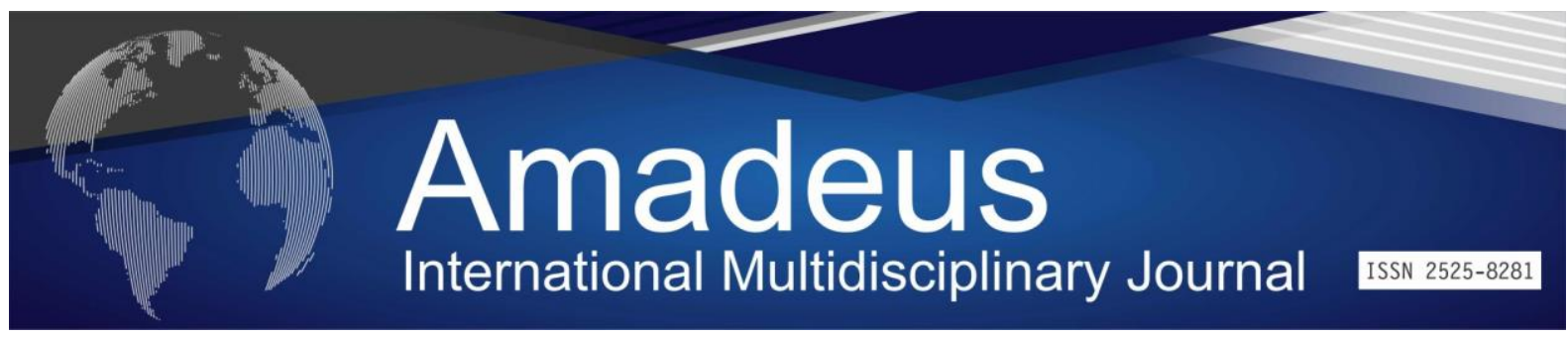

DOI: 10.14295/aimj.v4i8.102

\title{
Videolaparoscopic Treatment of Endometriosis: A Systematic Review
}

Whallyson Pinheiro

Mascarenhas ${ }^{1}$

Stenio Santos Moura ${ }^{2}$

Lucas Benevides Maia ${ }^{3}$,

Pedro Hugo Bezerra Maia

Filho ${ }^{4}$,

Pedro de Sousa Leite

Mondeyv de Freitas Pascoal ${ }^{6}$,

Francisco Felippe Araújo

Rolim $^{7}$

\begin{abstract}
Endometriosis is characterized by the presence of stroma and endometrial glands in extrauterine sites, which can compromise several sites. The definitive diagnosis of endometriosis is through laparotomy or laparoscopy. The objective of the present work is to analyze and evaluate the importance of laparoscopic treatment of endometriosis in the last 5 years. The present study is a systematic review of the literature, according to the Preferred Reporting Items for Systematic Reviews and Meta-Analyzes (PRISMA) methodology. Of the 386 articles found, only 14 were included. The articles used show that the use of videolaparoscopy in the treatment of endometriosis is effective and has its advantages, but with some complications. One of the main factors is the delay in indicating laparoscopy, which can lead to late diagnosis and impairment of the reproductive future of these women.
\end{abstract}

Keywords: Advantages, Laparoscopy, videolaparoscopy and endometriosis.

\section{Tratamento Videolaparoscópico da Endometriose: Uma Revisão Sistemática}

\begin{abstract}
Resumo: A endometriose é caracterizada pela presença de estroma e glândulas endometriais em locais extrauterinos, podendo comprometer diversos locais. $\mathrm{O}$ diagnóstico definitivo da endometriose é por meio da realização de laparotomia ou laparoscopia. O objetivo do presente trabalho é analisar e avaliar a importância do tratamento videolaparoscópico da endometriose nos últimos 5 anos. O presente estudo trata-se de uma revisão sistemática da literatura, conforme a metodologia Preferred Reporting Items for Systematic Reviews and Meta-Analyses (PRISMA). Dos 386 artigos encontrados, apenas 14 foram incluídos. Os artigos utilizados mostram que o uso de videolaparoscopia no tratamento de endometriose é eficaz e tem suas vantagens, porém algumas complicações. Um dos principais fatores é a demora na indicação da videolaparoscopia pode levar ao diagnóstico tardio e ao comprometimento do futuro reprodutivo dessas mulheres.
\end{abstract}

Descritores: Vantagens, Laparoscopia, videolaparoscopia e endometriose.

\footnotetext{
${ }^{1}$ Residente Cirurgia Geral- Hospital Regional do Cariri, Juazeiro do Norte-CE, Brasil, Whallyson10@ hotmail.com; ${ }^{2}$ Residente Cirurgia Geral- Hospital Regional do Cariri, Juazeiro do Norte-CE, Brasil, stenio_moura33@ @otmail.com;

${ }^{3}$ Residente Cirurgia Geral- Hospital Regional do Cariri, Juazeiro do Norte-CE, Brasil, benevides973@ hotmail.com;

${ }^{4}$ Residente Cirurgia Geral- Hospital Regional do Cariri, Juazeiro do Norte-CE, Brasil, pedrohugomaia@ hotmail.com;

${ }^{5}$ Graduando em Medicina pela Faculdade de Medicina Estácio de Juazeiro do Norte, CE, Juazeiro do Norte - Brasil, pedroed1913@hotmail.com;

${ }^{6}$ Cirurgião do Aparelho Digestivo e Coordenador da Residência de Cirurgia Geral do Hospital Regional do Cariri - HRC. Juazeiro do Norte, CE - Brasil, mondeyv@gmail.com;

${ }^{7}$ Cirurgião Geral e Professor da Residência de Cirurgia Geral no Hospital Regional do Cariri - HRC. Juazeiro do Norte, CE Brasil, felippe.rolim1@gmail.com
} 


\section{Introdução}

A endometriose é caracterizada pela presença de estroma e glândulas endometriais em locais extrauterinos, podendo comprometer diversos locais, entre eles os ovários, peritônio, ligamentos úteros-sacros, região retro cervical, septo retovaginal, além de bexiga, reto, sigmoide e outras porções do tubo digestivo (SCHENKEN, 2016). Vale salienta que é uma doença com grande morbidade e considerada o principal fator de risco para gravidez ectópica (Hwang et al., 2016).

A incidência da endometriose no mundo é de 5\% a $10 \%$ em mulheres em idade fértil, podendo chegar aproximadamente $50 \%$ naquelas com dor pélvica. No Brasil, a endometriose apresenta uma prevalência de 10 a $15 \%$ na população feminina fértil, e os custos da doença chegam a 10,4 milhões de reais por ano (Podgaec et al., 2014; Yela;Trigo;Benetti-Pinto, 2017).

Da Silva (2012) descreve que a endometriose pode causar inúmeras manifestações clínicas, apresentando quadros assintomáticos ou sintomáticos, com intensidade e localização variando de acordo o grau de acometimento da doença. De acordo com Culley e outros colaboradores (2013) a etiopatogenia ainda não está bem estabelecida, entretanto as evidências indicam que a combinação de fatores genéticos, hormonais e imunológicos poderia contribuir para a formação e o desenvolvimento dos focos ectópicos de endometriose.

Devido à dificuldade do diagnóstico e a complexidade da própria doença o tratamento da endometriose (clínico ou cirúrgico) permanece em discussão e tem como objetivo primário a redução da dor, a melhora da qualidade de vida e o aumento das chances de gestação destas mulheres (Fauconnier et al., 2013; Culley et al., 2013). Além disso, é importante compreender que o diagnóstico definitivo da endometriose é realizado através da laparotomia ou laparoscopia (Campos; Navalho; Cunha, 2008).

A videolaparoscopia ou laparoscopia com exérese da cápsula do cisto endometriótico é considerado o tratamento mais frequentemente realizado, com evidências na literatura de que possa resultar em menor taxa de recorrência da lesão (BOURDEL et al., 2011; SENGOKU et al., 2013; OUCHI et al., 2014). Além do que, esse método apresenta algumas vantagens, como tempo de recuperação pós-cirúrgico mais curto, menor perda sanguínea, menor dor pós-operatória e permanência no hospital (Navarro et al., 2006). 
Diante da elevada incidência dessa patologia na atualidade, o presente estudo tem como objetivo analisar e avaliar a importância do tratamento videolaparoscópico da endometriose nos últimos 5 anos.

\section{Metódo}

O presente trabalho trata-se de um estudo de revisão sistemática da literatura, conduzido conforme a metodologia Preferred Reporting Items for Systematic Reviews and Meta-Analyses - PRISMA (Moher et al., 2009). O objetivo da revisão sistemática é buscar o consenso sobre alguma temática especifica e sintetizar o conhecimento de uma dada área por meio da formulação de uma pergunta, identificação, seleção e avaliação crítica de estudos científicos contidos em bases de dados eletrônicas. A partir desse processo, ela permite, além de aprofundar o conhecimento sobre a temática investigada, apontar lacunas que precisam ser preenchidas por meio da realização de novas investigações (Munoz et al., 2002; Lopes; Francolli, 2008).

A coleta de dados do presente estudo se deu no período de setembro a outubro do ano de 2019. Para inclusão dos artigos, foi feita os seguintes passos:

- Busca de artigos nas bases eletrônicas Scientific Electronic Library Online (SciELO), Pubmed e BVS, por meio das palavras-chaves selecionadas segundo a classificação dos Descritores em Ciências da Saúde (DeCS): Tratamento, vídeolaparoscopia, laparoscopia, endometriose, doença.

- Para seleção dos artigos realizou-se, primeiramente, a leitura dos resumos das publicações selecionadas com o objetivo de refinar a amostra por meio de critérios de inclusão e exclusão.

- Foram incluídos artigos originais publicados nos últimos 5 anos (2014-2019), tanta na língua portuguesa como na inglesa.

Após a consulta às bases de dados e a aplicação das estratégias de busca, foram lidos todos os resumos resultantes. Nos casos em que a leitura do resumo não era suficiente para estabelecer se o artigo deveria ser incluído, considerando-se os critérios de inclusão definidos, o artigo foi lido na íntegra para determinar sua elegibilidade. Quando o resumo era suficiente, os artigos eram selecionados e então obtidos a versão integral para confirmação de elegibilidade e inclusão no estudo. 
Os critérios de exclusão foram: artigos de reflexão, estudos que apresentavam duplicidade entre as bases, ausência de resumo nas plataformas de busca on-line e de artigos que não eram completos, artigos que não tenha como foco principal tratamento videolaparoscópico de endometriose.

Por meio desse processo, a amostra final foi constituída por 14 artigos. A avaliação crítica dos artigos consistiu na leitura do estudo na integra e, consequentemente, na elaboração de quadros sinópticos com os dados coletados com informações de cada pesquisa, a saber: autores/data, objetivo da pesquisa, tipo de estudo, principais resultados e/ou conclusões (Munoz et al., 2002). Para uma maior compreensão foi feito o uso da técnica de analise temática de conteúdo por meio da leitura e releitura dos resultados dos estudos, procurando identificar aspectos relevantes que se repetiam ou se destacavam (Bardin, 1977).

\section{Resultados e Discussão}

A seleção inicial resultou em 386 estudos. Tendo como base a leitura do título e do resumo de cada artigo, 96 atenderam aos critérios de inclusão e 77 foram excluídos pelo fato de não bordarem o tema. Na leitura dos artigos na íntegra, 5 foram excluídos por não terem como objetivo principal o tratamento videoparoscópico da endometriose, conforme explanado no critério de exclusão. Dessa forma, a amostra final resultou composta por 14 artigos. As fases do processo para obtenção dos textos analisados na presente revisão estão demonstradas na Figura 1.

Figura 01: Fases do processo de obtenção dos artigos incluídos no estudo, Juazeiro do Norte-CE, 2019.

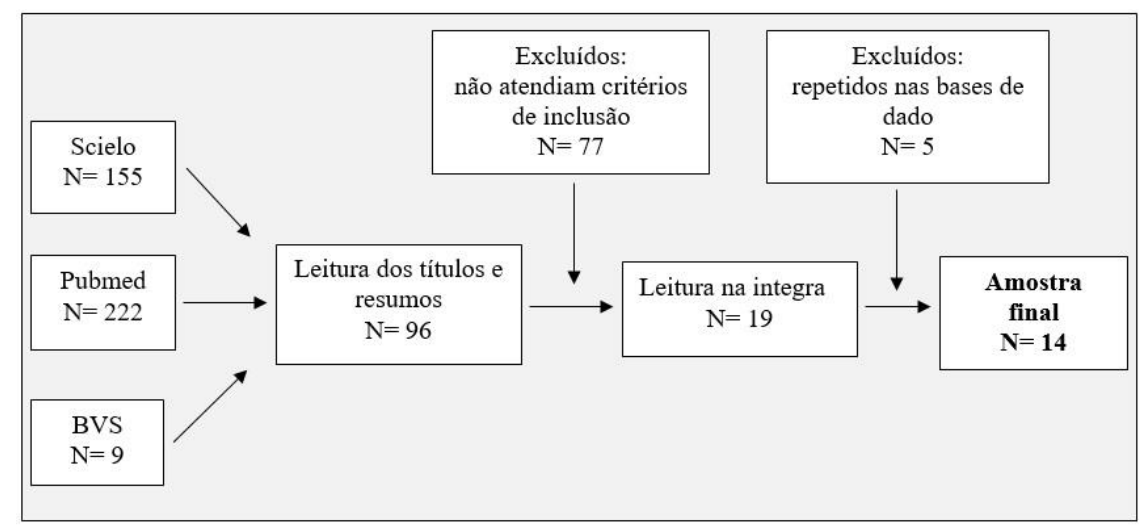

Fonte: Elaborado pelos autores, 2019. 
Após a leitura na íntegra dos 14 artigos iniciou-se a análise dos dados. Para facilitar a análise e apresentação dos resultados, elaborou-se uma tabela com dados sobre autor/ano, objetivo da pesquisa, tipo de estudo, principais achados (Tabela 1) Os dados estão organizados por ano de publicação.

Tabela 1 - Distribuição dos artigos incluídos na Revisão Sistemática. Juazeiro do Norte CE, 2019.

\begin{tabular}{|c|c|c|c|c|}
\hline Autores/Ano & Título & Objetivo & $\begin{array}{l}\text { Tipo de } \\
\text { estudo }\end{array}$ & Principais achados \\
\hline $\begin{array}{l}\text { Mettler; } \\
\text { Ruprai; } \\
\text { Alkatout, } \\
2014\end{array}$ & $\begin{array}{l}\text { Impact of medical and } \\
\text { Surgical Treatment of } \\
\text { Endometriosis on the } \\
\text { Cure of Endometriosis } \\
\text { and Pain }\end{array}$ & $\begin{array}{l}\text { Avaliar três estratégias } \\
\text { diferentes de tratamento } \\
\text { (medicação hormonal, } \\
\text { tratamento cirúrgico ou } \\
\text { combinado) e discute a } \\
\text { influência } \\
\text { endometriose na cura } \\
\text { desta doença e no alívio } \\
\text { da dor. }\end{array}$ & Estudo clínico & $\begin{array}{l}450 \text { pacientes } \\
\text { endometriose genital, com } \\
\text { idades entre } 18 \text { e } 44 \text { anos, } \\
\text { foram distribuídos } \\
\text { aleatoriamente em três grupos } \\
\text { de tratamento na primeira } \\
\text { laparoscopia. Eles foram } \\
\text { reavaliados em uma segunda } \\
\text { laparoscopia (D } 426 / 10 \text { ), um a } \\
\text { dois meses após as três terapias } \\
\text { hormonais de um mês para os } \\
\text { grupos } 1 \text { e } 3 \text { e cinco a seis } \\
\text { meses depois para o grupo } 2 \\
\text { (somente tratamento cirúrgico). }\end{array}$ \\
\hline $\begin{array}{l}\text { Kim et al., } \\
2014\end{array}$ & $\begin{array}{l}\text { Recurrence of ovarian } \\
\text { endometrioma after } \\
\text { second- } \\
\text { line,conservative, } \\
\text { laparoscopic } \\
\text { enucleation }\end{array}$ & $\begin{array}{l}\text { Avaliar a taxa de } \\
\text { recorrência cumulativa do } \\
\text { endometrioma após uma } \\
\text { enucleação de cisto endo- } \\
\text { metriótico conservador e } \\
\text { laparoscópico de segunda } \\
\text { linha e analisar os fatores } \\
\text { que influenciam a } \\
\text { ocorrência do } \\
\text { endometriom }\end{array}$ & & $\begin{array}{l}\text { No total, } 183 \text { pacientes foram } \\
\text { acompanhados por } 33,227,7 \\
\text { meses (intervalo, 6e121 meses). } \\
\text { Trinta e oito (20,8\%) pacientes } \\
\text { apresentaram recidiva após a } \\
\text { cirurgia de segunda linha e } 24 \\
(13,1 \%) \text { pacientes foram } \\
\text { submetidos a uma terceira } \\
\text { cirurgia. O tempo médio de } \\
\text { recorrência foi de } 24 \text { 3,36 } \\
\text { meses (MEV) (variação de } 3 \text { e } \\
72 \text { meses). As taxas de } \\
\text { recorrência cumulativa por } \\
\text { paciente aos 12, } 24,36 \text { e } 60 \\
\text { meses após a cirurgia de } \\
\text { segunda linha foram } 7,7 \% \text {, } \\
13,7 \% \text {, 21,3\% e } 37,5 \% \text {, } \\
\text { respectivamente. Após análise } \\
\text { multivariada e análise de } \\
\text { covariância, a pontuação e o } \\
\text { estágio revistos da American } \\
\text { Fertility Society foram } \\
\text { significativamente maiores em } \\
\text { pacientes que experimentaram } \\
\text { uma terceira recorrência de } \\
\text { endometrioma. }\end{array}$ \\
\hline
\end{tabular}

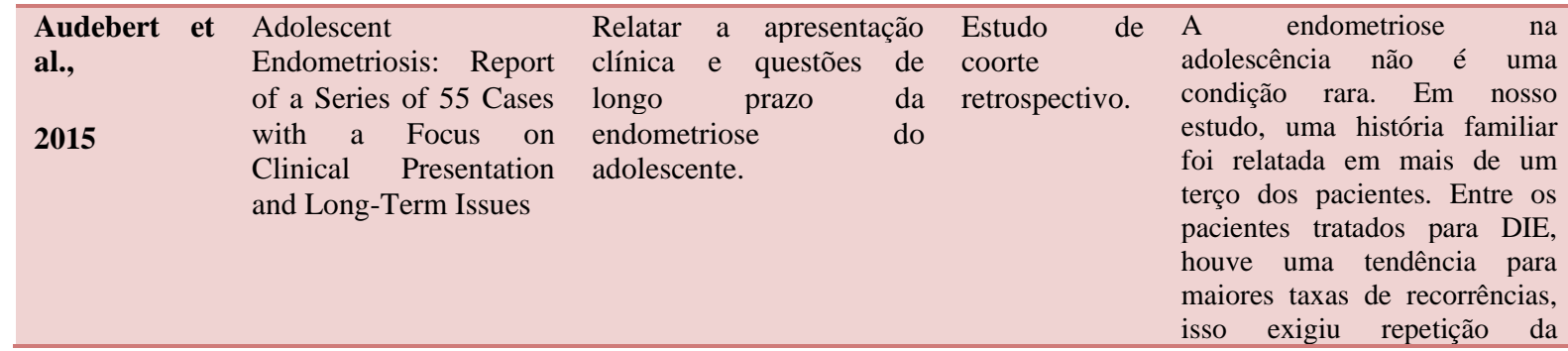




\begin{tabular}{|c|c|c|c|c|}
\hline & & & & $\begin{array}{l}\text { laparoscopia. No entanto, o } \\
\text { impacto na fertilidade } \\
\text { subsequente parecia ter sido } \\
\text { limitado. }\end{array}$ \\
\hline $\begin{array}{l}\text { Georgievska } \\
\text { et al., } \\
2015\end{array}$ & $\begin{array}{lr}\text { Effect of } & \text { Two } \\
\text { Laparoscopic } & \\
\text { Techniques } & \text { for } \\
\text { Treatment of } & \text { Ovarian } \\
\text { Endometrioma } & \text { on } \\
\text { Ovarian Reserve } & \end{array}$ & $\begin{array}{l}\text { Avaliar o efeito de duas } \\
\text { técnicas laparoscópicas no } \\
\text { tratamento de } \\
\text { endometriomas ovarianos } \\
\text { na reserva ovariana. }\end{array}$ & $\begin{array}{l}\text { Estudo } \\
\text { prospectivo }\end{array}$ & $\begin{array}{l}\text { No grupo A (operado com } \\
\text { cistectomia), o volume } \\
\text { ovariano foi de } 53,46 \pm 29,97 \\
\mathrm{~cm}^{3} \text { antes da cirurgia, que caiu } \\
\text { para } 13,06 \pm 7,34 \mathrm{~cm}^{3} \text { após um } \\
\text { mês e } 13,28 \pm 7,17 \mathrm{~cm}^{3} \text { após } \\
\text { três meses. A análise estatística } \\
\text { mostrou uma redução } \\
\text { significativa no volume } \\
\text { ovariano um e três meses após } \\
\text { a cirurgia (p } \leq 0,01 \text { ). No grupo B } \\
\text { (operado com punção e } \\
\text { endocoagulação), o volume } \\
\text { ovariano foi de } 58,34 \pm 37,99 \\
\mathrm{~cm}^{3} \text { antes da cirurgia, que caiu } \\
\text { para } 18,96 \pm 7,90 \mathrm{~cm}^{3} \text { em um } \\
\text { mês e } 17,38 \pm 6,86 \mathrm{~cm}^{3} \text { em três } \\
\text { meses após a cirurgia. }\end{array}$ \\
\hline
\end{tabular}

\begin{tabular}{|c|c|c|c|c|}
\hline $\begin{array}{l}\text { Posadzka et } \\
\text { al., } \\
2015\end{array}$ & $\begin{array}{l}\text { Treatment efficacy for } \\
\text { pain complaints in } \\
\text { women with } \\
\text { endometriosis of the } \\
\text { lesser pélvis after } \\
\text { laparoscopic } \\
\text { electroablation vs. } \mathrm{CO} 2 \\
\text { laser ablation }\end{array}$ & $\begin{array}{l}\text { Avaliar a eficácia do } \\
\text { tratamento laparoscópico } \\
\text { com o uso de ablação a } \\
\text { laser de CO2 vs. } \\
\text { eletroablação em relação } \\
\text { às queixas de dor nos } \\
\text { pacientes afetados }\end{array}$ & $\begin{array}{l}\text { Estudo } \\
\text { caso }\end{array}$ & $\begin{array}{l}\text { Os resultados do tratamento } \\
\text { laparoscópico foram } \\
\text { monitorados após } 3 \text { e } 6 \text { meses } \\
\text { de pós-operatório. Pacientes de } \\
\text { ambos os grupos relataram dor } \\
\text { menos intensiva antes/durante a } \\
\text { menstruação (dismenorreia) } 6 \\
\text { meses no pós-operatório, com } \\
\text { tendência mais distinta no } \\
\text { grupo de eletroablação } \\
\text { (p=0,004) em comparação ao } \\
\text { grupo de ablação a laser (p }= \\
0,025 \text { ). Apesar da melhora } \\
\text { inicial relatada no exame de } 3 \\
\text { meses (p = 0,008), } 6 \text { meses } \\
\text { após a cirurgia, foi observado } \\
\text { um aumento estatisticamente } \\
\text { significativo na intensidade da } \\
\text { dor nos dois grupos. }\end{array}$ \\
\hline $\begin{array}{l}\text { Ruan } \\
\text { Liang, ; } \\
\text { Huang, 2015 }\end{array}$ & $\begin{array}{l}\text { Analysis of laparoscopy } \\
\text { on endometriosis } \\
\text { patients with high } \\
\text { expression of CA } 125\end{array}$ & $\begin{array}{lr}\text { Analisar o efeito clínico } \\
\text { da laparoscopia em } \\
\text { pacientes } & \text { com } \\
\text { endometriose e alta } \\
\text { expressão de CA125. }\end{array}$ & $\begin{array}{l}\text { Estudo } \\
\text { caso }\end{array}$ & $\begin{array}{l}\text { A cirurgia laparoscópica em } \\
\text { pacientes com endometriose } \\
\text { com alta expressão de CA125 é } \\
\text { curativa e a recuperação dos } \\
\text { pacientes após a cirurgia é } \\
\text { promissora. }\end{array}$ \\
\hline
\end{tabular}

\begin{tabular}{|c|c|c|c|c|}
\hline $\begin{array}{l}\text { Sousa et al., } \\
2015\end{array}$ & $\begin{array}{l}\text { Tratamentos na } \\
\text { Endometriose: Uma } \\
\text { revisão sistemática }\end{array}$ & $\begin{array}{l}\text { Analisar quais tratamentos } \\
\text { vem sendo utilizados até } \\
\text { então para endometriose e } \\
\text { qual seu grau de } \\
\text { evidência. }\end{array}$ & $\begin{array}{l}\text { Revisão } \\
\text { sistemática }\end{array}$ & $\begin{array}{l}\text { Para dor pélvica, são descritos } \\
\text { os tratamentos empíricos } \\
\text { baseados em orientações físicas } \\
\text { e aconselhamentos, } \\
\text { progestágenos, } \\
\text { anticoncepcionais, } \\
\text { antiinflamatórios e SIU-LNG } \\
\text { (dispositivo de liberação } \\
\text { hormonal intra-útero). Já os } \\
\text { tratamentos citados para } \\
\text { infertilidade são, ablação } \\
\text { cirúrgica das lesões, cirurgias } \\
\text { mais complexas que envolvem } \\
\text { outros sistemas além do } \\
\text { reprodutor, tratamento } \\
\text { hormonal pós cirúrgicos e } \\
\text { inseminação intra-útero e }\end{array}$ \\
\hline
\end{tabular}




\begin{tabular}{|c|c|c|c|c|}
\hline & & & & fertilização in vitro. \\
\hline $\begin{array}{l}\text { Yela et al., } \\
2016\end{array}$ & $\begin{array}{l}\text { Ovarian Endometrioma: } \\
\text { Rate of Recurrence and } \\
\text { Factors Correlated }\end{array}$ & $\begin{array}{l}\text { Avaliar a taxa de } \\
\text { recorrência } \\
\text { endometrioma ovariano } \\
\text { durante um período de } \\
\text { cinco anos e os fatores de } \\
\text { risco associado a esse } \\
\text { distúrbio. }\end{array}$ & $\begin{array}{l}\text { Estudo } \\
\text { transversal } \\
\text { retrospectivo }\end{array}$ & $\begin{array}{l}\text { A taxa de recorrência do } \\
\text { endometrioma foi de } 40,2 \% \text { e o } \\
\text { tempo médio de recorrência da } \\
\text { doença foi de } 34 \text { meses após a } \\
\text { cirurgia. Houve uma associação } \\
\text { significativa entre uma maior } \\
\text { taxa de recorrência do } \\
\text { endometrioma e a presença de } \\
\text { outros focos }(p=0,03) \text {. Não } \\
\text { houve significância estatística } \\
\text { da recorrência do } \\
\text { endometrioma com as demais } \\
\text { variáveis. }\end{array}$ \\
\hline
\end{tabular}

\begin{tabular}{|ll}
\hline Byrne et al., & $\begin{array}{l}\text { Laparoscopic excision } \\
\text { of deep rectovaginal }\end{array}$ \\
endometriosis in BSGE \\
endometriosis centres: a \\
multicentre prospective \\
cohort study
\end{tabular}

Estimar a eficácia e a Um estudo de segurança da excisão coorte cirúrgica laparoscópica da endometriose retovaginal. prospectivo e multicêntrico
Aos 6 meses após a cirurgia, houve reduções significativas na dor pélvica pré-menstrual, menstrual e não cíclica, dispareunia profunda, disquezia, lombalgia e dor na bexiga. Além disso, houve reduções significativas na dificuldade de esvaziar, frequência intestinal, urgência, esvaziamento incompleto, constipação e passagem de sangue. Essas reduções foram mantidas em 2 anos, com exceção da dificuldade de anulação. A qualidade de vida global melhorou significativamente de uma pontuação média de prétratamento de 55/100 para 80/100 em 6 meses.

\section{Centini et al. Effect of Anterior Avaliar o desempenho Compartment Endometriosis Excision \\ reprodutivo desfechos de \\ pacientes \\ com \\ estudo retrospectivo} on Infertility infertilidade inexplicável submetida a cirurgia para endometriose infiltrativa profunda

(DIE)

envolvendo

compartimento anterior. multicêntrico

Os sintomas relacionados à endometriose da bexiga foram resolvidos em $84,2 \%$ dos casos, com uma taxa de recorrência de $1,8 \%$ no seguimento de 2 anos, sem necessidade de cirurgia adicional.

\begin{tabular}{|c|c|c|c|c|}
\hline $\begin{array}{l}\text { Nogueira et } \\
\text { al., } \\
2018\end{array}$ & $\begin{array}{l}\text { Tratamento da } \\
\text { endometriose pélvica: } \\
\text { uma revisão sistemática }\end{array}$ & $\begin{array}{l}\text { Esclarecer os tratamentos } \\
\text { usados atualmente para a } \\
\text { endometriose. }\end{array}$ & $\begin{array}{l}\text { Revisão } \\
\text { integrativa }\end{array}$ & $\begin{array}{l}\text { O tratamento cirúrgico pode ser } \\
\text { radical e conservador. } \\
\text { Atualmente, o uso da } \\
\text { laparoscopia tem demonstrado } \\
\text { boa eficácia, porque permite } \\
\text { uma excelente observação da } \\
\text { pelve e da destruição das } \\
\text { lesões. }\end{array}$ \\
\hline Gomes et al., & $\begin{array}{lr}\text { Ressecção } & \text { de } \\
\text { Endometriose Ureteral } \\
\text { com Reimplante } \\
\text { Uretero-Vesical por } \\
\text { Videolaparoscopia }\end{array}$ & $\begin{array}{l}\text { Relatar um caso de } \\
\text { endometriose ureteral com } \\
\text { hidronefrose } \\
\text { com abordada } \\
\text { ressecção e reimplante } \\
\text { uretero-vesical r por } \\
\text { videolaparoscopia. }\end{array}$ & Relato de caso & $\begin{array}{l}\text { A paciente recebeu alta } \\
\text { hospitalar no } 4^{\circ} \text { dia de pós- } \\
\text { operatório. A análise } \\
\text { histopatológica das peças, } \\
\text { revelou adenomiose no corpo } \\
\text { uterino, cervicite crônica no } \\
\text { colo uterino, endometriose de } \\
\text { ureter e ovários com cistos } \\
\text { folicular e hemorrágico. A } \\
\text { paciente retornou com } 1 \\
\text { semana, } 2 \text { meses e } 1 \text { ano de }\end{array}$ \\
\hline
\end{tabular}


pós-operatório, com melhora completa do quadro aqui descrito e não sendo identificado complicações.

\begin{tabular}{|c|c|c|c|c|}
\hline Pang et al., & $\begin{array}{l}\text { Endometriosis on the } \\
\text { surface of the uterus } \\
\text { mimicking a malignant } \\
\text { tumor }\end{array}$ & $\begin{array}{l}\text { Conscientizar sobre a } \\
\text { endometriose na } \\
\text { superfície do útero, } \\
\text { simulando tumor maligno } \\
\text { e, assim, gerenciá-lo com } \\
\text { precisão }\end{array}$ & $\begin{array}{l}\text { Relato de caso } \\
\text { com revisão } \\
\text { de literatura }\end{array}$ & $\begin{array}{l}\text { A recuperação do paciente } \\
\text { ocorreu sem intercorrências. } \\
\text { Três meses após a operação, o } \\
\text { paciente apresentava bom } \\
\text { estado geral sem dor periódica } \\
\text { e o nível de CA } 125 \text { voltou ao } \\
\text { normal. }\end{array}$ \\
\hline Sima et al., & $\begin{array}{l}\text { Novel diagnosis of } \\
\text { mesenteric } \\
\text { endometrioma }\end{array}$ & $\begin{array}{l}\text { Relatar endometrioma } \\
\text { mesentérico }\end{array}$ & $\begin{array}{l}\text { Estudo } \\
\text { caso }\end{array}$ & $\begin{array}{l}\text { O resultado do paciente foi } \\
\text { favorável, com alta às } 72 \text { horas } \\
\text { após a cirurgia. O relato } \\
\text { histopatológico revelou que os } \\
\text { cistos mesentéricos e ovarianos } \\
\text { eram endometriomas. }\end{array}$ \\
\hline
\end{tabular}

Fonte: Elaborado pelos autores, 2019

Os artigos incluídos nessa revisão analisaram o tratamento com videolaparoscopia de endometriose.

Durante anos, houve um debate entre ginecologistas, qual era a melhor técnica laparoscópica para operação de pacientes com endometriose ovariana (Georgievska et al., 2015). De acordo com Nogueira e outros colaboradores (2018) a laparoscopia apresenta algumas vantagens, como tempo de recuperação pós-cirúrgico mais curto, menor perda sanguínea, menor dor pós-operatória e permanência no hospital. No entanto, eles ainda falam que o tratamento da endometriose pélvica é factível, e pode ser tanto medicamentoso, quanto cirúrgico, ou ainda a associação de ambos, antes ou após a cirurgia, sendo que é necessário levar em consideração fatores como a gravidade dos sintomas, o desejo de gestar, a extensão e localização da doença, a idade da paciente, os efeitos adversos dos medicamentos, as taxas de complicações cirúrgicas e seus custos.

Um estudo comparativo de duas técnicas de videolaparoscopia identificaram que a reserva ovariana diminui após a cirurgia laparoscópica usando as duas técnicas laparoscópicas. Porém, essa redução foi mais frequênte com cistectomia em comparação com a ablação do cisto endometriótico (Georgievska et al., 2015).

Para o diagnostico de endometriose a videolaparoscopia tem sido usado com bastante frequência. No trabalho de Sima e outros colaboradores (2019) os autores afirmam que através da laparoscopia foi possível confirmar o diagnóstico de endometriose ovariana e peritoneal. 
Em relação à eficácia da videolaparoscopia Centini e outros colaboradores (2018) discorrem que a excisão laparoscópica da endometriose do compartimento anterior é eficaz na restauração da fertilidade em pacientes com infertilidade inexplicável e em outros tratamentos de sintomas relacionados à endometriose. Diante disse, os autores Nácul e Spritzer (2010) falam que apesar dos exames de imagem apresentarem uma acurácia boa no diagnóstico da endometriose, a videolaparoscopia com biópsia das lesões para análise histopatológica ainda é o padrão-ouro no diagnóstico da endometriose. Além disso, Ruan, Liang e Huang (2015) abordam que pacientes com endometriose com alta expressão de CA125 a cirurgia é curativa e a recuperação de pacientes após a cirurgia produz bons resultados.

De acordo com Audebert e outros colaboradores (2015) o uso de videolaparoscopia no tratamento de pacientes que apresentam endometriose de infiltração profunda pode haver algumas recorrências (sintomas, lesões) e talvez necessitem de novas laparoscopias, porém o impacto disso sobre a fertilidade subsequente é considerado limitado.

Byrne e outros colaboradores (2018) abordam que a excisão cirúrgica laparoscópica parece ser eficaz no tratamento da dor pélvica e sintomas intestinais, melhorando a qualidade de vida relacionada à saúde e apresenta baixa taxa de complicações graves quando realizada em centros especializados. Em um estudo de caso a abordagem videolaparoscópica foi bem indicada e efetiva no caso, sendo que houve a ressecção completa dos focos endometrióticos. Nesse estudo eles relatam as vantagens dessa técnica versus a laparotômica, uma vez que a videolaparoscopia inclui menor morbidade, menor tempo de internação e dor no pós-operatório, bem como e estética da cicatriz cirúrgica (Gomes et al., 2018).

De acordo com Mettler e outros colaboradores (2003) para uma abordagem adequada da endometriose é necessário o uso de três passos: laparoscopia cirúrgica com remoção de todos focos visíveis de endometriose, terapia endócrina de 3 a 6 meses, e uma laparoscopia subsequente com ressecção focos residuais, adesiólise e cirurgia reconstrutiva de os órgãos. Entretanto para Mettler, Ruprai e Alkatout (2014) o tratamento combinado é a o tratamento mais bem sucedido para endometriose. A comparação das três estratégias terapêuticas diferentes implica um benefício mais alto para o tratamento combinado, na medida em que o estadiamento e a redução dos sintomas (período sem doença) e as taxas de gravidez. 
Em uma revisão sistemática os autores avaliaram os tratamentos para endometriose em que os resultados mostram que em casos mais graves de endometriose onde a dor é aguda e a infertilidade está presente, a laparoscopia é utilizada como padrão ouro de tratamento, no entanto esta é paliativa e não tem garantia total de eficácia (Sousa et al., 2015).

Apesar da maioria dos autores relatarem as vantagens do uso de vídeolaparoscopia, alguns autores relatam que apesar das vantagens desse tratamento, existe algumas complicações. Os autores Kim e outros colaboradores (2014) falam que a recorrência dos endometriomas ovarianos após tratamento videolaparoscópico ainda permanece elevada e sua prevenção incerta porque a patogênese da recidiva ainda é desconhecida.

Vale ressaltar que é importante que tanto os ginecologistas quanto os oncologistas estejam cientes de que a endometriose pode simular malignidade ovariana. A tomografia por emissão de pósitrons antes da cirurgia, a biópsia multiponta e a análise frozensection durante a cirurgia podem aumentar a precisão do diagnóstico e tratamento (Pang et al., 2019).

\section{Considerações Finais}

Atualmente o tratamento padrão é a laparoscopia. A videolaparoscopia apresenta inúmeras vantagens como menor dor no pós-operatório, menor permanência hospitalar, menor taxa de complicação pós-cirúrgica. Ainda a muito a ser desvendado sobre a endometriose para que então identifique uma terapia eficaz na minimização dos sintomas e com o mínimo de efeitos colaterais.

$\mathrm{Na}$ presente revisão foi possível ver as vantagens do uso de videolaparoscopia no tratamento de endometriose, é uma técnica minimamente invasiva, porém existem algumas complicações após a cirurgia.

Infelizmente o tratamento com videolaparoscopia não cura, mas podem amenizar as manifestações parcialmente ou completo nas pacientes. Em algumas circunstâncias a doença pode voltar mesmo com a histerectomia. A cirurgia laparoscópica pode favorecer no aumento da fertilidade e a chance de sucesso dependendo da gravidade da endometriose. 
São necessários mais trabalhos, principalmente de estudo de caso para poder desvendar sobre a endometriose a fim de eleger um tratamento eficaz na minimização dos sintomas e com o mínimo de efeitos colaterais.

\section{Referências}

Audebert, Alain et al. (2015). Adolescent endometriosis: report of a series of 55 cases with a focus on clinical presentation and long-term issues. Journal of minimally invasive gynecology, v. 22, n. 5, p. 834-840.

Bardin. L. (1997). Análise de conteúdo. Lisboa: Editora Edições 70.

Bourdel, N. et al. (2011). Surgery for the management of ovarian endometriomas: from the physiopathology to the pre-, peri-and postoperative treatment. Gynecologie, obstetrique \& fertilite, v. 39, n. 12, p. 709-721.

Byrne, Dominic et al. (2018). Laparoscopic excision of deep rectovaginal endometriosis in BSGE endometriosis centres: a multicentre prospective cohort study. BMJ open, v. 8, n. 4, p. e018924.

Campos, Cláudia; Navalho, Márcio; Cunha, Teresa Margarida.(2008). Endometrioseepidemiologia, fisiopatologia e revisão clínica e radiológica. Acta Radiológica Portuguesa, v. 20 , n. 80 , p. $67-77$.

Centini, Gabriele et al. (2018). Effect of Anterior Compartment Endometriosis Excision on Infertility. JSLS: Journal of the Society of Laparoendoscopic Surgeons, v. 22, n. 4.

Culley, Lorraine et al.(2013). The social and psychological impact of endometriosis on women's lives: a critical narrative review. Human reproduction update, v. 19, n. 6, p. 625639.

Da Silva, Ana Daniela Rodrigues Lima. (2012). Endometriose e infertilidade: o papel do tratamento cirúrgico prévio a ciclos de procriação medicamente assistida. Instituto De Ciências Biomédicas Abel Salazar. Universidade do Porto. Portugal.

Fauconnier, Arnaud et al. (2013). Comparison of patient-and physician-based descriptions of symptoms of endometriosis: a qualitative study. Human reproduction, v. 28, n. 10, p. 2686-2694.

Georgievska, Jadranka et al. (2015). Effect of two laparoscopic techniques for treatment of ovarian endometrioma on ovarian reserve. Medical Archives, v. 69, n. 2, p. 88.

Gomes, José Walter Feitosa et al. (2018). Ressecção de Endometriose Ureteral com Reimplante Uretero-Vesical por Videolaparoscopia. Revista Urominas, v. 60015, p. 061. 
Hwang, Alan et al. (2016). Risk factors for ectopic pregnancy in the Taiwanese population: a retrospective observational study. Archives of gynecology and obstetrics, v. 294, n. 4, p. 779-783.

Kim, Mi-La et al. (2014). Recurrence of ovarian endometrioma after second-line, conservative, laparoscopic cyst enucleation. American journal of obstetrics and gynecology, v. 210, n. 3, p. 216. e1-216. e6.

Lopes, Ana Lúcia Mendes; Fracolli, Lislaine Aparecida. (2008). Revisão sistemática de literatura e metassíntese qualitativa: considerações sobre sua aplicação na pesquisa em enfermagem. Texto \& Contexto-Enfermagem, v. 17, n. 4, p. 771-778.

Mettler, L. et al.(2003). Accuracy of laparoscopic diagnosis of endometriosis. JSLS: Journal of the Society of Laparoendoscopic Surgeons, v. 7, n. 1, p. 15.

Mettler, Liselotte; Ruprai, R.; Alkatout, Ibrahim. (2014). Impact of medical and surgical treatment of endometriosis on the cure of endometriosis and pain. BioMed research international, v. 2014.

Moher, David et al. (2009). Preferred reporting items for systematic reviews and metaanalyses: the PRISMA statement. Annals of internal medicine, v. 151, n. 4, p. 264-269.

NACUL, Andrea Prestes; SPRITZER, Poli Mara. (2010). Aspectos atuais do diagnóstico e tratamento da endometriose. Rev. Bras. Ginecol. Obstet. Rio de Janeiro, v. 32, n. 6, p. 298307.

Navarro, Paula Andrea de Albuquerque Salles et al. (2006). Tratamento da endometriose. Revista Brasileira de ginecologia e obstetrícia, v. 28, n. 10, p. 612-623.

Nogueira ACR, et al. (2018). Tratamento da endometriose pélvica: uma revisão sistemática. Revista Científica FAGOC-Saúde, v. 3, n. 2, p. 38-43.

Ouchi, Nozomi et al. (2014). Recurrence of ovarian endometrioma after laparoscopic excision: risk factors and prevention. Journal of Obstetrics and Gynaecology Research, v. 40, n. 1, p. 230-236.

Pang, Lihong et al. (2019). Endometriosis on the surface of the uterus mimicking a malignant tumor: A case report with literature review. Medicine, v. 98, n. 21.

Podgaec, S. et al. (2014). Manual de endometriose. São Paulo: Federação Brasileira das Associações de Ginecologia e Obstetrícia (FEBRASGO).

Posadzka, Ewa et al. (2015). Treatment efficacy for pain complaints in women with endometriosis of the lesser pelvis after laparoscopic electroablation vs. CO 2 laser ablation. Lasers in medical science, v. 30, n. 1, p. 147-152.

Ruan, Y. Q.; Liang, W. G.; Huang, S. H. (2015). Analysis of laparoscopy on endometriosis patients with high expression of CA125. Eur Rev Med Pharmacol Sci, v. 19, n. 8, p. 13347.

Schenken, Robert S. (2016). Endometriosis: Pathogenesis, clinical features, and diagnosis. Waltham, MA: Walters Kluwer Health. 
Munoz, Susana Inés et al. (2002). Revisão sistemática de literatura e metanálise: noções básicas sobre seu desenho, interpretação e aplicação na área da saúde. In: Proceedings of the 8. Brazilian Nursing Communication Symposium.

Sengoku, Kazuo et al. (2013). Clinicopathologic risk factors for recurrence of ovarian endometrioma following laparoscopic cystectomy. Acta obstetricia et gynecologica Scandinavica, v. 92, n. 3, p. 278-284.

Sima, Romina-Marina et al. (2019). Novel diagnosis of mesenteric endometrioma: Case report. Medicine, v. 98, n. 29.

Sousa, Tatiane Regina et al. (2015). Tratamentos na Endometriose: Uma revisão sistemática. ConScientiae Saúde, v. 14, n. 4, p. 655-664.

Yela, Daniela Angerame; Trigo, Lucas; Benetti-Pinto, Cristina Laguna. (2017). Evaluation of cases of abdominal wall endometriosis at Universidade Estadual deCampinas in a period of 10 years. Revista Brasileira de Ginecologia e Obstetrícia/RBGO Gynecology and Obstetrics, v. 39, n. 08, p. 403-407.

\section{How to cite this article (APA format):}

Mascarenhas, Whallyson Pinheiro; Moura, Stenio Santos; Maia, Lucas Benevides; Maia Filho, Pedro Hugo Bezerra; Leite, Pedro de Sousa; Pascoal, Mondeyv de Freitas; Rolim, Francisco Felippe Araújo (2020). Videolaparoscopic Treatment of Endometriosis: A Systematic Review. Am. In. Mult. J., February to May. (8) 5, 25-37.

Received: 02/16/2020;

Accepted: 02/19/2020. 